\title{
Molecular Characterization of Annexin B2, B3 and B12 in Taenia multiceps
}

\author{
Cheng Guo ${ }^{1,+}$, Yue Xie ${ }^{1,+}$, Yuchen Liu ${ }^{1}$, Ning Wang ${ }^{1}$, Jiafei Zhan ${ }^{1}$, Xuan Zhou ${ }^{2}$, \\ Christiana Angel ${ }^{1,3}{ }^{\circ}$, Xiaobin Gu ${ }^{1}$, Weimin Lai ${ }^{1}$, Xuerong Peng ${ }^{4}$ and Guangyou Yang ${ }^{1, *}$ \\ 1 Department of Parasitology, College of Veterinary Medicine, Sichuan Agricultural University, \\ Chengdu 611130, China; Chengguo_92@hotmail.com (C.G.); xyue1985@gmail.com (Y.X.); \\ liuyuchen1229@163.com (Y.L.); wangningzhuhui@hotmail.com (N.W.); zhanjiafei@hotmail.com (J.Z.); \\ qazi5502@yahoo.com (C.A.); guxiaobin198225@126.com (X.G.); aliciaheml@hotmail.com (W.L.) \\ 2 Institute of Animal Genetics and Breeding, College of Animal Science and Technology, \\ Sichuan Agricultural University, Chengdu 611130, China; zhouxuan198866@163.com \\ 3 Department of Veterinary Parasitology, Faculty of Veterinary Sciences, Shaheed Benazir Bhutto University of \\ Veterinary and Animal Sciences, Sindh 67210, Pakistan \\ 4 Department of Chemistry, College of Life and Basic Science, Sichuan Agricultural University, \\ Chengdu 611130, China; pxuerong@aliyun.com \\ * Correspondence: guangyouyang@sicau.edu.cn; Tel.: +86-189-8055-8366 \\ + These authors contributed equally to the work.
}

Received: 23 October 2018; Accepted: 12 November 2018; Published: 19 November 2018

\begin{abstract}
Coenurus cerebralis, the metacestode of Taenia multiceps, causes coenurosis, a disease severely affecting goat, sheep, cattle and yak farming and resulting in huge economic losses annually. Annexins bind calcium ions and play an important role in flatworm parasite development. To explore potential functions of annexins in T. multiceps, three homologous genes, namely, TmAnxB2, TmAnxB3 and TmAnxB12, were screened from the transcriptome dataset, amplified from C. cerebralis cDNA and subjected to bioinformatics analysis. Then, polyclonal antibodies recognizing the recombinant TmAnxB2 (rTmAnxB2) and rTmAnxB3 were prepared for localization of TmAnxB2 and TmAnxB3 in different tissues and developmental stages by immunofluorescence. The transcription of all three genes was also measured by relative fluorescent quantitative PCR. The sizes of rTmAnxB2, rTmAnxB3 and rTmAnxB12 were 58.00, 53.06 and $53.51 \mathrm{kDa}$, respectively, and rTmAnxB12 was unstable. Both rTmAnxB2 and rTmAnxB3 were recognized by goat-positive T. multiceps sera in Western blots. Immunofluorescence revealed that TmAnxB2 and TmAnxB3 were localized in the protoscolex and cyst wall and TmAnxB3 was also detected in adult cortex. TmAnxB2 and TmAnxB12 mRNA levels were determined to be highest in oncospheres and protoscolex, whereas transcription of TmAnxB3 was highest in scolex and immature segments. Taken together, these findings indicate that TmAnxB2 and TmAnxB12 may play critical roles in T. multiceps larvae, while TmAnxB3 may have important functions in adults. These results will lay the foundation for functional research of annexins in T. multiceps.
\end{abstract}

Keywords: Taenia multiceps; annexins; prokaryotic expression; immunofluorescence localization; qRT-PCR

\section{Introduction}

Taenia multiceps is a flatworm parasite that is widely distributed around the world, and its eggs enter the environment via feces of its definitive hosts such as dogs, wolves, foxes and other canids [1-3]. Intermediate hosts, such as goats, sheep, cattle and yaks, are infected with coenurosis following ingestion of pasture containing parasite eggs. Metacestodes of T. multiceps, known as Coenurus cerebralis, 
then parasitize the central nervous system and intermuscular, subcutaneous, and liver tissues of these intermediate hosts [4-6] and cause clinical manifestations such as circling symptoms, depression and even death $[7,8]$, which lead to serious economic losses to farming, especially in economically developing countries within Africa and Southeast Asia [9-11]. Furthermore, this parasite also causes zoonotic infections in humans and poses severe threats to public health [12-14].

Annexins are a class of phosphatide binding proteins with calcium binding activity. These proteins are widely expressed in all eukaryotes and play important roles in various biological activities such as cell membrane transport, signal transduction, and calcium channel formation $[15,16]$. Furthermore, these proteins have been known to regulate the biological responses related to inflammation and help to maintain the stability of biofilm structure [15-17]. In parasites, annexins are thought to have important functions in maintaining cell structural integrity and regulating parasites to host immune responses [18]. However, there is very little information available on annexins in the parasitic cestode T. multiceps. In the present study, based on available transcriptome data of adult T. multiceps that were

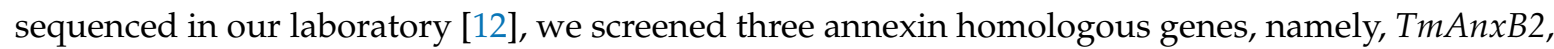
$T m A n x B 3$ and $T m A n x B 12$, and further amplified and expressed these three recombinant proteins TmAnxB2 (rTmAnxB2), rTmAnxB3 and rTmAnxB12 using a prokaryotic expression system. Due to instability of the rTmAnxB12, only TmAnxB2 and TmAnxB3 proteins were investigated for their localization in different tissues and developmental stages of T. multiceps by immunofluorescence. Meanwhile, the transcriptional differences of all three genes were also analyzed by the relative fluorescent quantitative PCR. These findings will lay a foundation for the future functional analysis of annexins in T. multiceps.

\section{Materials and Methods}

\subsection{Ethical Approval}

This study was reviewed and approved by the Animal Ethics Committee of Sichuan Agricultural University (AECSCAU; Approval No. 2011-028). Animals were handled strictly in accordance with the animal protection law of the People's Republic of China (released on 18 September 2009) and the National Standards for Laboratory Animals in China (executed on 1 May 2002).

\subsection{Parasites and Animals}

Coenuri larvae were isolated from the brains of naturally infected goats at a slaughterhouse in Sichuan Province, China. Adult worms were derived from a four-month-old beagle dog infected with C. cerebralis cysts at 45 days post-infection. The dog was kept alone in a clean cage, with clean food and water supplied ad libitum. Gravid proglottids from dog feces were collected before sacrifice. T. multiceps oncospheres were obtained as previously described [19]. All parasite materials were washed four times with sterile saline and stored in liquid nitrogen or $4 \%$ paraformaldehyde. In addition, T. multiceps-positive and negative goat serum samples were provided by the Department of Parasitology, College of Veterinary Medicine, Sichuan Agricultural University (Sichuan, China).

\subsection{Bioinformatics Analysis}

The open reading frames (ORFs) of genes encoding TmAnxB2, TmAnxB3 and TmAnxB12 were predicted using ORF finder with 300-bp minimal ORF in length and "ATG only" of ORF start codon (http:/ / www.ncbi.nlm.nih.gov/gorf/gorf.html). Basic physicochemical properties and stability coefficients of these annexins were predicted by ProtParam with default parameters (http://web.expasy.org/protparam/), and signal peptides were assessed using SignalP (http:// www.cbs.dtu.dk/Services/SignalP/). BaCelLo (http://gpcr.biocomp.unibo.it/bacello/pred.htm) was used to predict the subcellular localization of the three proteins, and the protein secondary was predicted using the NPSA_server (http://npsa-pbil.ibcp.fr/). Tertiary (3D) structures were modeled through SWISS-MODEL (http://swissmodel.expasy.org/) and by referring to the $2.5 \AA$ 
resolution crystal structure of Schistosoma mansoni annexin (PDB accession No. 4MDV) for TmAnxB2, $1.42 \AA$ crystal structure of annexin V (PDB accession No. 1yii.1.A) for TmAnxB3 and $2.8 \AA$ crystal structure of annexin XII (PDB accession No. 1aei.1.A) for TmAnxB12. Phosphorylation sites were predicted by Motif Scan with default parameters (https://myhits.isb-sib.ch/cgi-bin/motif_scan), and phylogenetic analysis was conducted using maximum-likelihood (ML) method and plotted by PhyML 3.1 (http:/ / www.atgc-montpellier.fr/phyml/versions.php) using the Jones-Taylor-Thornton (JTT) model selected under the AIC criterion by ProtTest 3 (http:/ / darwin.uvigo.es/our-software/). Support for nodes was estimated by analyzing 1000 bootstrap replicates for each locus.

\subsection{Cloning, Expression and Purification of Recombinant TmAnxB2, TmAnxB3 and TmAnxB12}

Total RNA was extracted from oncospheres, coenuri, scolex, immature proglottids, mature proglottids, and gravid proglottids using an RNA Extraction Kit (Tiangen, Beijing, China) according to the manufacturer's instructions, and template cDNAs were synthesized using a RevertAid First Strand cDNA Synthesis Kit (Fermentas, ON, Canada). Based on the assembled T. multiceps transcriptome dataset (Unigene 18519) and the AnxB2 sequence of Taenia solium (GenBank accession No. AY998562.1), specific primers for TmAnxB2 were designed. Primers for TmAnxB3 were designed based on the T. multiceps transcriptome dataset (Unigene 19512) and the AnxB3 sequence of T. solium (GenBank accession No. DQ010543.1). Gene-specific primers for TmAnxB12 were designed using the T. multiceps transcriptome dataset (Unigene 17682) and an annexin from Echinococcus granulosus (GenDB No. EgrG_000237700). All three pairs of primers are listed in Table 1. Notably, apart from these three TmAnxBs, no other homologs were found within such T. multiceps transcriptome. After PCR amplifications, the products were digested with restriction enzymes, gel-purified and sub-cloned into the pET32a $(+)$ expression vector, and the resultant constructs were transformed into competent Escherichia coli BL21 (DE3) cells. E. coli cells containing pET32a-TmAnxB2, pET32a-TmAnxB3 and pET32a-TmAnxB12, respectively, were cultivated at $37{ }^{\circ} \mathrm{C}$ for $4 \mathrm{~h}$, induced with $1 \mathrm{mM}$ isopropyl- $\beta$-D-1-thiogalactopyranoside (IPTG), and cultured for further $6 \mathrm{~h}$. Then, the recombinant TmAnxB2 (rTmAnxB2), rTmAnxB3 or rTmAnxB12 with a $\approx 18-\mathrm{kDa}$ His-tag protein was harvested and purified using Ni2+ affinity chromatography with NGC 10 system (Bio-Rad Laboratories, Inc., Hercules, USA). Protein purity was analyzed by $12 \%$ sodium dodecyl sulphate-polyacrylamide gel electrophoresis (SDS-PAGE).

Table 1. Primers used for PCR amplification of Taenia multiceps annexins.

\begin{tabular}{clcc}
\hline \multirow{2}{*}{ Genes } & \multicolumn{1}{c}{ Primers } & Restriction Enzyme Sites & Reference Sequences \\
\hline \multirow{2}{*}{ TmAnxB2 } & F: CGCGGATCCATGGCAAAAAATACTCGCTC & BamHI & Unigene18519 \\
& R: CCGCTCGAGTTAGGATTCATTCAGTAGTGCG & XhoI & Ts AY998562.1 \\
\hline \multirow{2}{*}{ TmAnxB3 } & F: CCGGAATTCATGGCGACTGTCAAGCTTT & EcoRI & Unigene19512 \\
& R: CCGCTCGAGTCACTCCAGTATGGTGAGCA & XhoI & Ts DQ010543.1 \\
\hline \multirow{2}{*}{ TmAnxB12 } & F: CGCGGATCCATGAATGGGCGTCCAACTA & BamHI & Unigene17682 \\
& R: CCGGAATTCTTATGCATTCCCCTCTACAA & EcoRI & EgrG_000237700 \\
\hline
\end{tabular}

Note: Black and bold italics signify restriction enzyme sites.

\subsection{Western Blotting}

Given that the purified rTmAnxB12 was unstable, only rTmAnxB2 and rTmAnxB3 were included in the Western blotting and subsequent immunohistochemical localization. Along with total worm extract, rTmAnxB2 and rTmAnxB3 were separated by 12\% SDS-PAGE and transferred onto a nitrocellulose (NC) filter membrane (Bio-Rad). Membranes were washed three times with TRIS-buffered saline Tween-20 buffer, blocked with $5 \%(w / v)$ skim milk solution in phosphate-buffered saline (PBS) for $2 \mathrm{~h}$ at room temperature, and T. multiceps-positive goat serum (1:100 $v / v$ dilution in PBS buffer) was added and incubated overnight at $4{ }^{\circ} \mathrm{C}$. After three washes, horseradish peroxidase (HRP)-conjugated rabbit anti-goat IgG (Bio-Rad) diluted in PBS (1:3000 $v / v$ dilution) was added and 
incubated at room temperature for $2 \mathrm{~h}$. After four washes, blots were visualized using an Enhanced HRP-DAB Chromogenic Substrate Kit (Tiangen) according to the instructions.

\subsection{Fluorescence Immunohistochemistry}

Fresh worm tissues including oncospheres, coenuri, scolex, immature proglottids, mature proglottids, and gravid proglottids of T. multiceps were embedded in paraffin and sectioned at $5 \mu \mathrm{m}$ thickness. After deparaffinization, slices were placed in citrate buffer $(0.01 \mathrm{M}, \mathrm{pH} 6.0)$ at $95^{\circ} \mathrm{C}$ for 15 min. After three washes with PBS buffer $(0.01 \mathrm{M}), 3 \% \mathrm{H}_{2} \mathrm{O}_{2}$ was added to the slices and incubated at $37{ }^{\circ} \mathrm{C}$ for $30 \mathrm{~min}$. After three washes, rabbit anti-rTmAnxB2 or anti-TmAnxB3 IgG as well as naïve rabbit serum diluted with PBS $(1: 100 \mathrm{v} / \mathrm{v})$ were added to slices and incubated at $4{ }^{\circ} \mathrm{C}$ for $14 \mathrm{~h}$. Fluorescein isothiocyanate (FITC)-conjugated goat anti-rabbit IgG diluted 1:100 in 0.1\% Evans Blue was then incubated with the slices at $37^{\circ} \mathrm{C}$ in a dark environment for $1 \mathrm{~h}$. After washing with PBS, stained samples were viewed under an Olympus BX50 fluorescence microscope (Olympus, Tokyo, Japan) using the red light as its sole light source.

\subsection{Quantitative Real-Time PCR}

Expression profiles of these three homologous TmAnxB2, TmAnxB3 and TmAnxB12 genes in oncospheres, protoscolex, scolex, immature proglottids, mature proglottids, and gravid proglottids were probed using quantitative real-time PCR (qRT-PCR). The actin gene was simultaneously used as an internal control for normalization. The qRT-PCR was performed using a CFX manager (Bio-Rad) according to the manufacturer's instructions. The cycling conditions comprised a pre-incubation at $95{ }^{\circ} \mathrm{C}$ for $30 \mathrm{~s}$, followed by 40 cycles at $95^{\circ} \mathrm{C}$ for $5 \mathrm{~s}, 57.4{ }^{\circ} \mathrm{C} / 55.7^{\circ} \mathrm{C}$ for $20 \mathrm{~s}$, and $72{ }^{\circ} \mathrm{C}$ for $20 \mathrm{~s}$. The primer sequences are provided in Table 2 . Relative expression values were calculated using the $2^{-\Delta \Delta C t}$ method. Each gene was tested in quadruplicate.

Table 2. Primers used for quantitative real-time PCR (qRT-PCR) amplification of T. multiceps annexins.

\begin{tabular}{|c|c|c|}
\hline Gene & Primers & Size (bp) \\
\hline TmAnxB2 & $\begin{array}{l}\text { F: GGTTCAACACGCCGTAGACAGAC } \\
\text { R: TGAGGACTCGCATGAGGAGGAAG }\end{array}$ & 101 \\
\hline TmAnxB3 & $\begin{array}{l}\text { F: TGCACCGCCACCAACAACG } \\
\text { R: CACTCGCTTGAAGTCGCCAGAG }\end{array}$ & 114 \\
\hline TmAnxB12 & $\begin{array}{l}\text { F: AGGAGGTGACGGAGATGAAGGC } \\
\text { R: GCAACGGCGATGATCTGGATGAG }\end{array}$ & 94 \\
\hline Actin & $\begin{array}{l}\text { F: CTAAGGCGAACCGTGAGAAGATGAC } \\
\text { R: GGCATGAGGCAAGGCGTAACC }\end{array}$ & 188 \\
\hline
\end{tabular}

\section{Results}

\subsection{Molecular Features of Taenia multiceps Annexins}

Sequencing analysis showed that genes for TmAnxB2, TmAnxB3 and TmAnxB12 contained ORFs of 1065 bp, $933 \mathrm{bp}$ and $957 \mathrm{bp}$, respectively, encoding predicted polypeptides of 354, 310 and 318 amino acids, respectively. None of the annexins showed signal peptides or transmembrane domains, however, the signature sequence K-G-X-G-T was observed in all these three annexins. The predicted molecular weights of TmAnxB2, TmAnxB3 and TmAnxB12 were 40.00, 35.06 and $35.51 \mathrm{kDa}$, respectively, and the predicted isoelectric points (pIs) were 6.42, 5.53 and 6.14, respectively. In addition, the stability of these proteins was also predicted, and the results showed that a higher instability index (40.05) was observed in TmAnxB12, indicating the relative instability of this protein. Similarity comparison of three annexins revealed a sequence similarity of $27.89-42.77 \%$ at the amino acid level. Multiple sequence alignment revealed that TmAnxB2 shares 96.33\% identity with AnxB2 from T. solium (TsAnxB2; GenBank accession No. AAY17503.1), 86.46\% with E. granulosus annexin 
(GenBank accession No. CDS21833.1), and 85.88\% with Echinococcus multilocularis annexin (GenBank accession No. CDI98110.1). TmAnxB3 shares 89.03\% identity with AnxB3 from T. solium (TsAnxB3; GenBank accession No. AAY27744.1), 61.94-80.65\% identity with other Taeniidae annexins, and only $38.71 \%$ identity with S. mansoni annexin (GenBank accession No. AAC79802.3). TmAnxB12 shares more than $85 \%$ identity with its orthologs in E. granulosus and E. multilocularis, and $43.22-43.99 \%$ identity with the equivalent proteins in Trematoda (Figure 1).

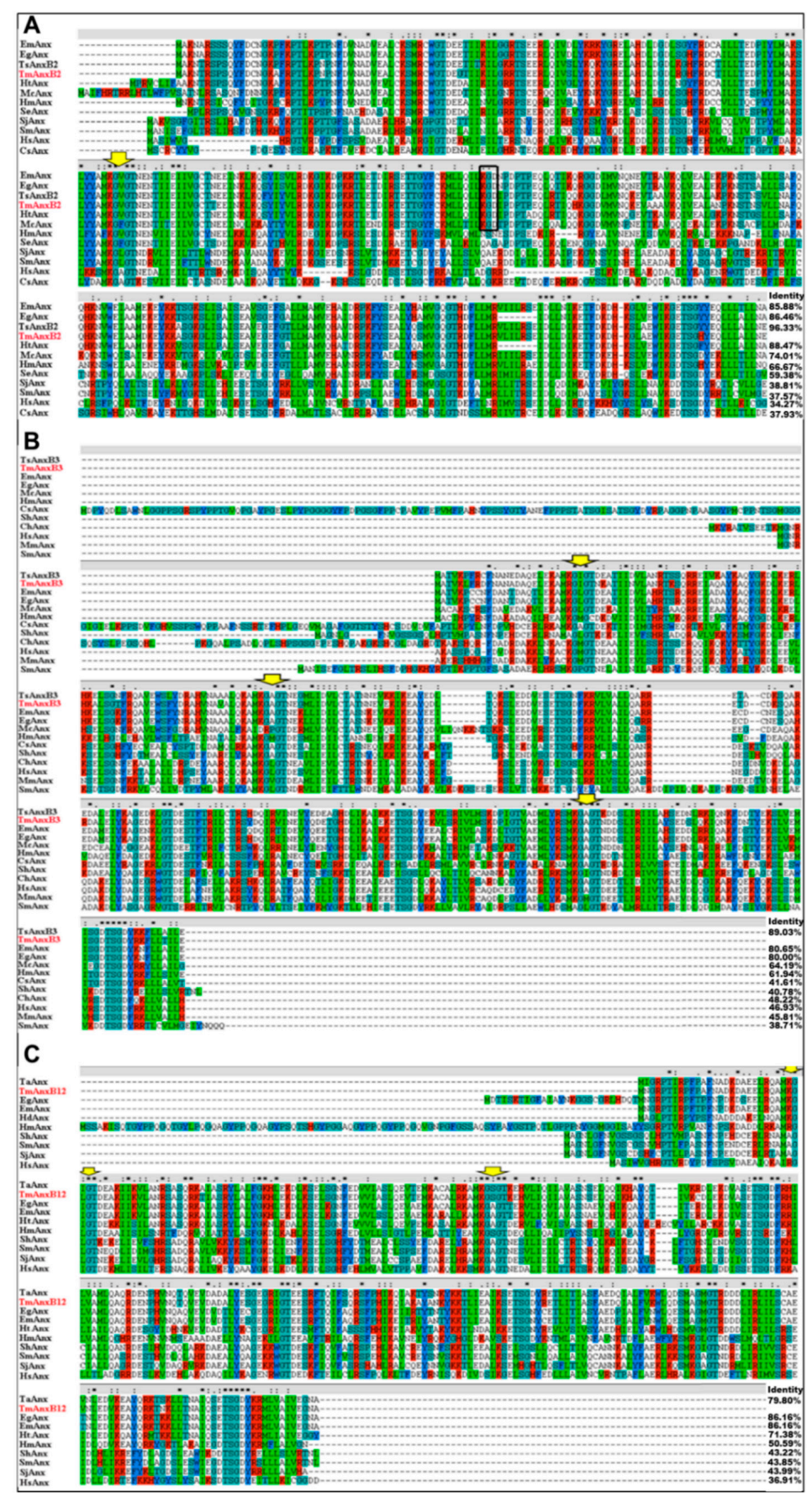

Figure 1. Sequence alignment analyses of: AnxB2 (A); AnxB3 (B); and AnxB12 (C) in T. multiceps and other species. (A) The following sequences were retrieved from the GenBank protein sequence database (accession numbers are indicated in parentheses): Em, Echinococcus multilocularis (CDI98110.1); Eg, Echinococcus granulosus (CDS21833.1); Ts, Taenia solium (AAY17503.1); Ht, Hydatigena taeniaeformis (A0A0R3X1T1); Mc, Mesocestoides corti (A0A0R3UMC3); Hm, Hymenolepis microstoma (CDS30725.1); 
Se, Spirometra erinaceieuropaei (ADM26238.1); Sj, Schistosoma japonicum (AAW25344.1); Sm, Schistosoma mansoni (AAC79802.3); Hs, Homo sapiens (AAH00871.1); Cs, Clonorchis sinensis(GAA48684.1); (B) The following sequences were retrieved from the GenBank protein sequence database (accession numbers are indicated in parentheses): Ts, Taenia solium (AAY27744.1); Em, Echinococcus multilocularis (CDI98572.1); Eg, Echinococcus granulosus (EUB59082.1); Mc, Mesocestoides corti (A0A0R3UJ25); Hm, Hymenolepis microstoma (CDS27549.1); Cs, C. sinensis (GAA33818.2); Sh, Schistosoma haematobium (KGB40261.1); Ch, Capra hicus (XP_013824596.1); Hs, Homo sapiens (CAG46637.1); Mm, Mus musculus (NP_081487.1); Sm, S. mansoni (AAC79802.3); (C) The following sequences were retrieved from the GenBank protein sequence database (accession numbers are indicated in parentheses): Ta, Taenia asiatica (A0A0R3W340); Eg, E. granulosus (EUB63408.1); Em, Echinococcus multilocularis (CDI98522.1); Hd, Hymenolepis diminuta (A0A0R3SPK4); Hm, Hymenolepis microstoma(CDS26802.2); Sh, Schistosoma haematobium (XP_012800019.1); Sm, S. mansoni (XP_018651719.1); Sj, Schistosoma japonicum (CAX69693.1); $\mathrm{Hs}$, Homo sapiens (AAH00871.1). The yellow arrow in the figure represents the K-G-X-G-T sequence; the black box is the KGD sequence.

Based on sequence alignment of multiple annexins from Taeniidae and Trematoda, a ML phylogenetic tree was constructed, in which three annexins of T. multiceps were located on different branches. Specifically, TmAnxB2 and TmAnxB3 showed relatively close relationships to annexin B2 and B3 of T. solium, respectively; however, TmAnxB12 was more closely related to orthologs in Echinococcus spp. and Hymenolepis microstoma (Figure 2).

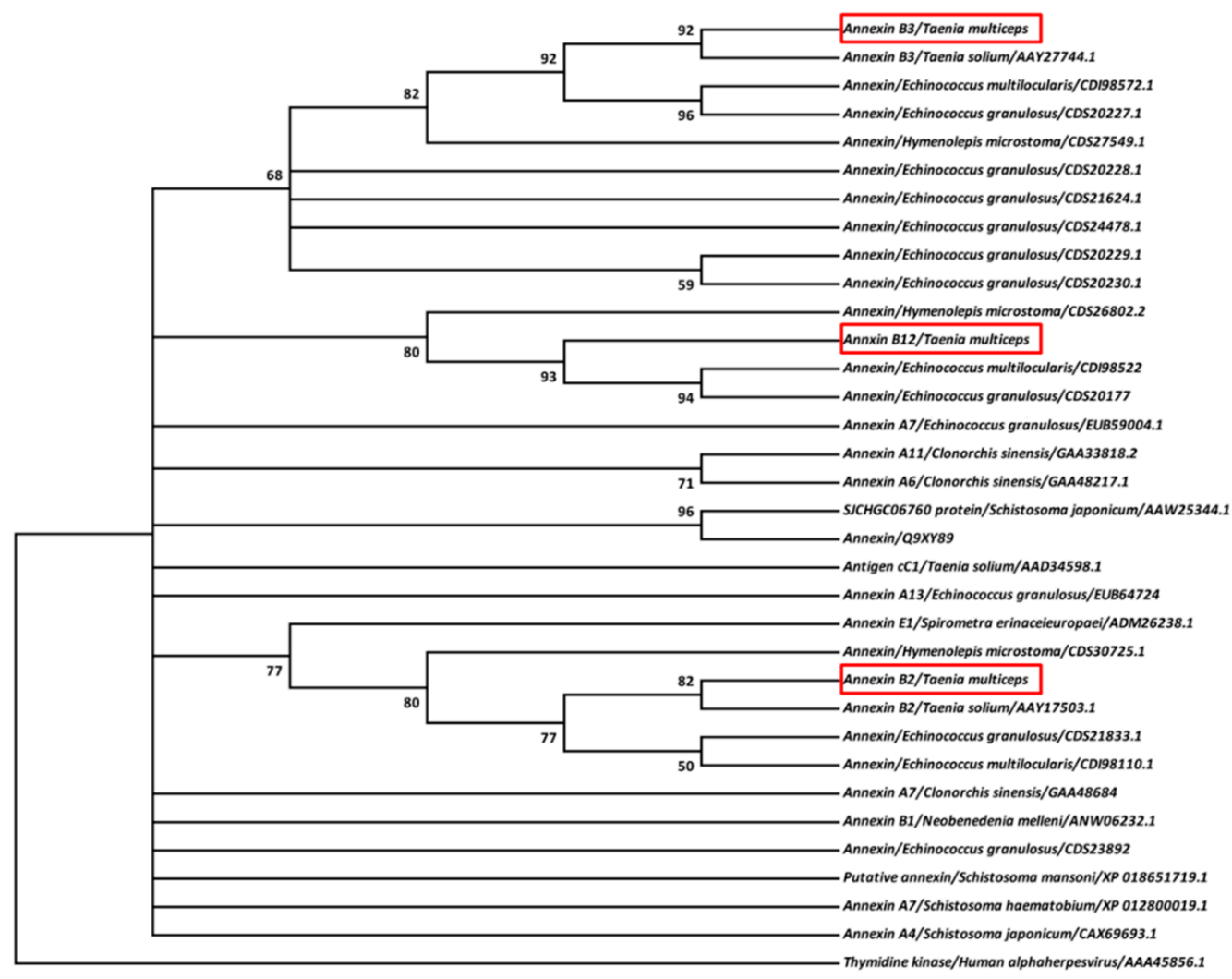

Figure 2. Phylogenetic analysis of annexin proteins. Phylogenetic analysis of the full-length amino acid sequences of T. multiceps annexins (in red boxes) and their homologs. The tree was constructed by the maximum-likelihood (ML) method and plotted with PhyML 3.1 with the Jones-Taylor-Thornton model selected by ProtTest 3 (http:/ / darwin.uvigo.es/our-software/). Bootstrap values are indicated at the nodes (1000 replications). The scale indicates an estimate of substitutions per site, using the optimized model setting. 


\subsection{Structural Analysis of Taenia multiceps Annexins}

Using the SWISS-MODEL database, TmAnxB2, TmAnxB3 and TmAnxB12 were modeled, and their predicted tertiary structures are depicted in Figure 3. Structural analysis revealed a common repeated alpha-helical domain. In addition, a unique $\alpha$-helical element between repeats II and III was observed in the 3D structure of TmAnxB2 (purple box in Figure 3A), but not in TmAnxB3 or TmAnxB12. Further analysis revealed that both TmAnxB3 and TmAnxB12 have calcium binding sites in domains III and I, respectively, unlike TmAnxB2. Specifically, a type II calcium ion binding motif (K-G-X-G-T-D-E-38 amino acid residues-D/E) was found in the repeat domain III of TmAnxB3, and a type III calcium binding motif (K-G-X-G-T-D-E) was observed in the repeat domain I of TmAnxB12 (grey boxes in Figure 3B,C), while a KGD motif was found between repeat domains II and III of TmAnxB2 instead of a calcium ion binding region. These observations therefore highlight that the structures of TmAnxB3 and TmAnxB12 are more similar to each other than to TmAnxB2.
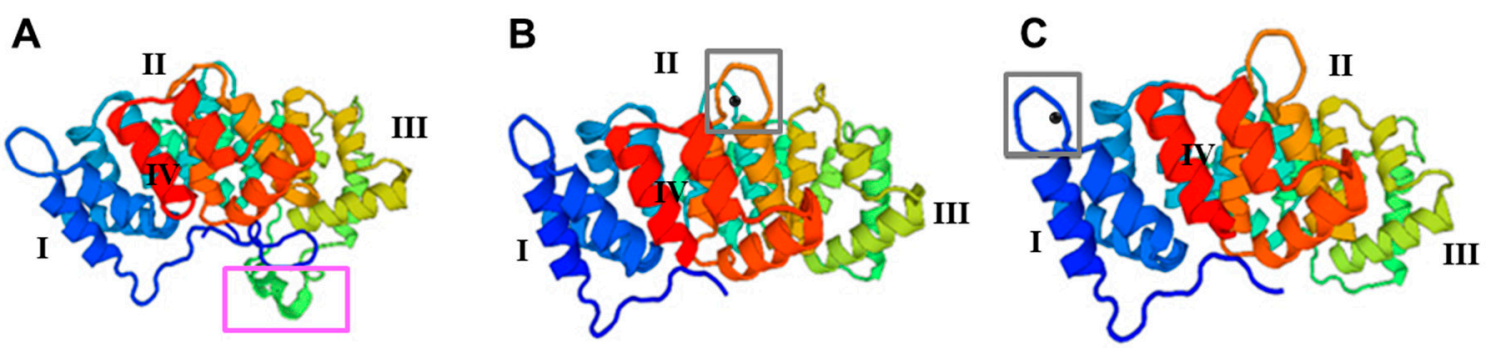

Figure 3. Predicted 3D structural models of: TmAnxB2 (A); TmAnxB3 (B); and TmAnxB12 (C). (A) The structure of TmAnxB2 is based on the crystal structure of $S$. mansoni Annexin (PDB accession code 4mdu1.A); (B) The structure of TmAnxB3 is based on the crystal structure of chicken annexin V (PDB accession code 1yii.1.A); (C) The structure of TmAnxB12 is based on the crystal structure of annexin XII (PDB accession code 1aei.1.A). The repeats in core domains of three molecules are coloured blue (repeat I), cyan (repeat II), yellow (repeat III) and red (repeat IV). The N-terminal domains are coloured sapphire blue, and the I/II linker regions are shown in sea green, the II/III linker in green and the III/IV linker in orange. Black spheres indicate the calcium ions.

TmAnxB2, TmAnxB3 and TmAnxB12 display typical characteristics of members of the annexin family, and have four repetitive domains of 57-72 amino acids. TmAnxB2 also has one tyrosine kinase phosphorylation site, two cAMP- and cGMP-dependent protein kinase phosphorylation sites, three protein kinase $\mathrm{C}$ phosphorylation sites, and four casein kinase II phosphorylation sites. TmAnxB3 has one cAMP- and cGMP-dependent protein kinase phosphorylation site, one tyrosine kinase phosphorylation site, six protein kinase $C$ phosphorylation sites, and twelve casein kinase II phosphorylation sites. TmAnxB12 has four protein kinase $C$ phosphorylation sites, 10 casein kinase II phosphorylation sites, and one protease weight complex region. Furthermore, both TmAnxB3 and TmAnxB12 have an EF-hand calcium ion binding region (grey boxes in Figure 3B,C) that is absent in TmAnxB2.

\subsection{Expression, Purification and Western Blotting of Taenia multiceps Annexins}

Recombinant rTmAnxB2, rTmAnxB3 and rTmAnxB12 were expressed with a $\approx 18$ kDa His-tag, but rTmAnxB12 was unstable and readily degraded, consistent with the ProtParam prediction. Therefore, subsequent immunolocalization and Western blotting experiments were carried out only on rTmAnxB2 and rTmAnxB3. The molecular masses of rTmAnxB2 and rTmAnxB3 were $\approx 58 \mathrm{kDa}$ and $53 \mathrm{kDa}$, respectively, close to the expected values. Rabbit anti-rTmAnxB2 and anti-rTmAnxB3 antibodies were prepared using purified rTmAnxB2 and rTmAnxB3, respectively. The native TmAnxB2 protein in adults was recognized using the rabbit anti-rTmAnxB2 antibody, yielding a single band of $\approx 40 \mathrm{kDa}$ that was absent in the negative control (Figure 4). Likewise, the native TmAnxB3 protein in adults was recognized by the rabbit anti-rTmAnxB3 antibody, yielding a single band of $\approx 35 \mathrm{kDa}$ (Figure 5). 
Furthermore, immunoblotting showed that both $r \operatorname{TmAnxB2}$ and $r \operatorname{TmAnxB3}$ were identified by T. multiceps-positive goat sera, suggesting the strong immunogenicity and immunoreactivity for both rTmAnxB2 and rTmAnxB3.

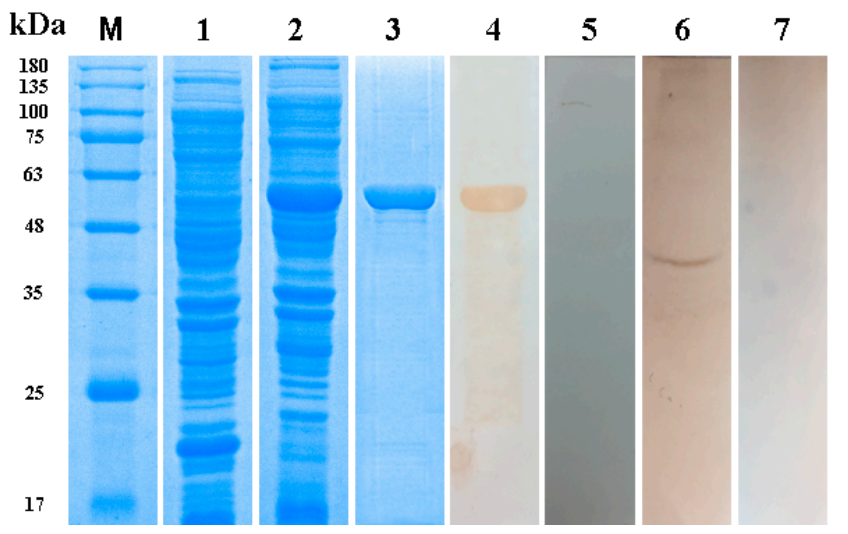

Figure 4. Purification and Western blotting of recombinant rTmAnxB2. M, Protein markers; 1, pET32a-vector; 2, pET32a-TmAnxB2; 3, Purified rTmAnxB2; 4, rTmAnxB2 detected by T. multiceps-positive goat serum; 5, rTmAnxB2 detected by T. multiceps-negative goat serum; 6 , Western blot of T. multiceps extracts detected by rabbit anti-rTmAnxB2-IgG; 7, Western blot of T. multiceps extracts detected by healthy rabbit serum (controls).

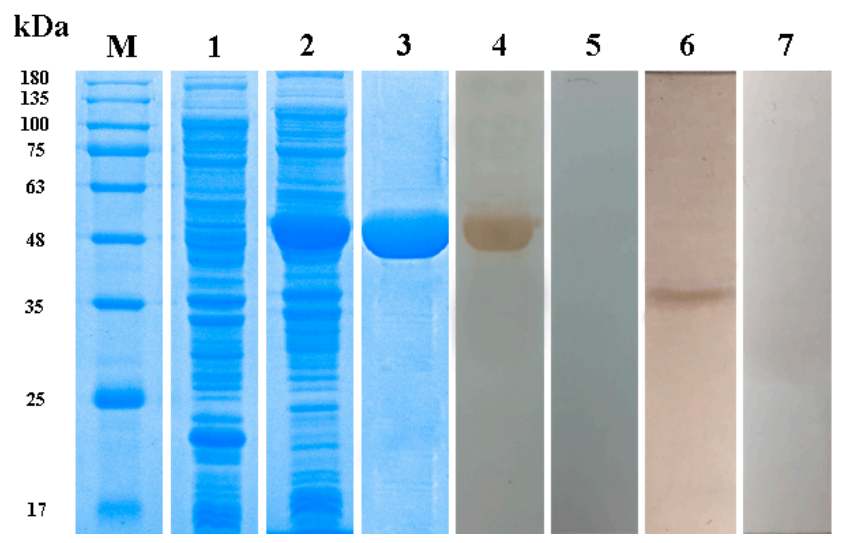

Figure 5. Purification and Western blotting of recombinant rTmAnxB3. M, Protein markers; 1, pET32a-vector; 2, pET32a-TmAnxB3; 3, Purified rTmAnxB3; 4, rTmAnxB3 detected by T. multiceps-positive goat serum; 5, rTmAnxB3 detected by T. multiceps-negative goat serum; 6: Western blot of T. multiceps extracts detected by rabbit anti-rTmAnxB3-IgG; 7, Western blot of T. multiceps extracts detected by healthy rabbit serum.

\subsection{Immunolocalization of $T m A n x B 2$ and $T m A n x B 3$}

Immunohistochemical analysis showed that TmAnxB2 and TmAnxB3 were distributed in the hooks of protoscolex and the germinal layers of the cyst wall of protoscolex (Figures 6 and 7). However, compared to TmAnxB2, a larger amount of TmAnxB3 was present in the cyst wall of protoscolex. Notably, no fluorescence signal was detected for TmAnxB2 in the scolex, neck, immature proglottids, or mature proglottids of adults; however, a weak fluorescence signal was detected in the eggs of gravid proglottids (Figure 6). Conversely, a strong fluorescence signal was observed for TmAnxB3 in all segments of adults, indicating the evidence of tissue specificity. For instance, TmAnxB3 was highly localized in the tegument zone (TZ) of adults, widely distributed in the parenchymatous zone (PZ) and sucker of scolex, and present in all areas of the neck (Figure 7). 


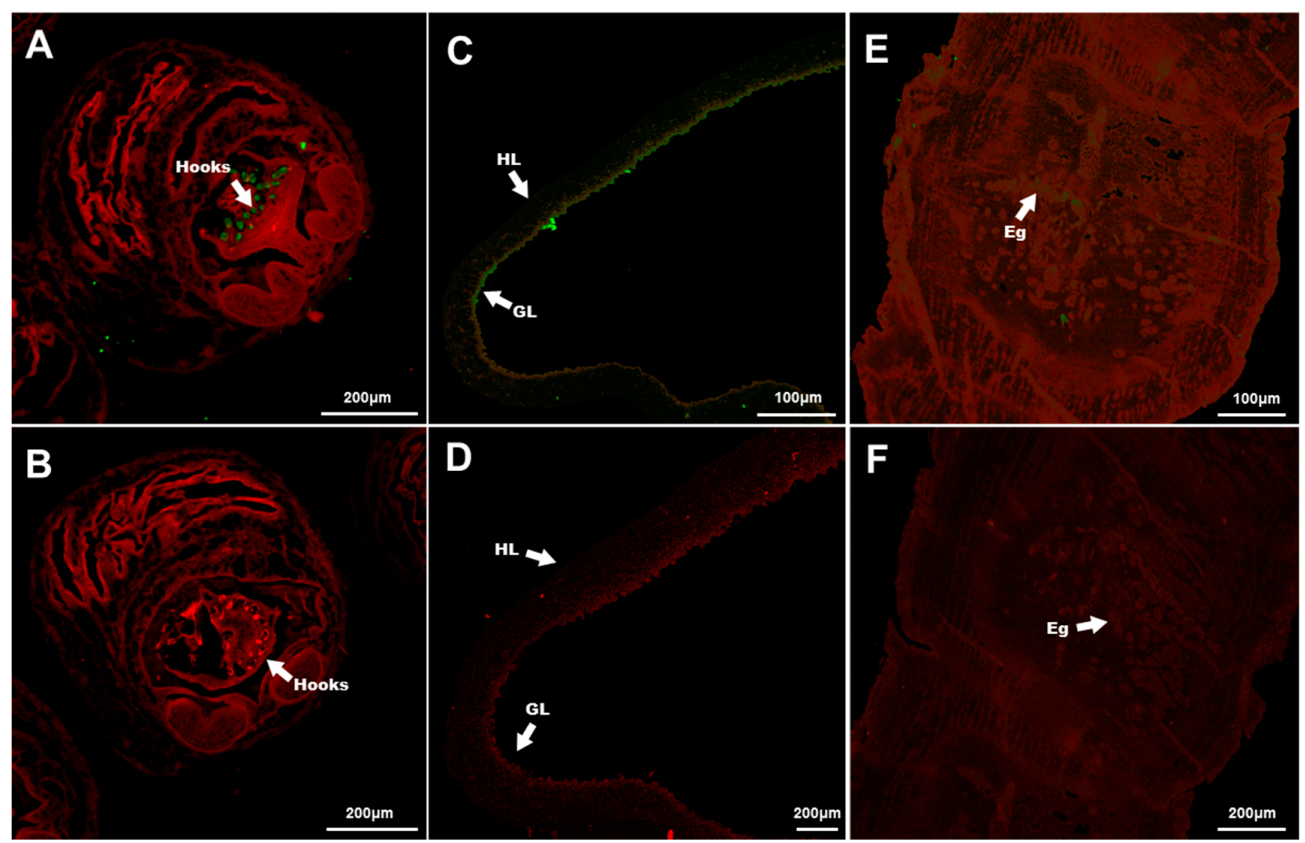

Figure 6. Immunofluorescence localization of TmAnxB2 in T. multiceps. Against the red background, the green fluorescent color shows the location of the native TmAnxB2 protein: (A) protoscolex with positive serum; (B) protoscolex with negative serum; (C) cyst wall with positive serum; (D) cyst wall with negative serum; (E) gravid proglottids with positive serum; and (F) gravid proglottids with negative serum; GL, germinal layer; HL, horny layer; Eg, eggs.

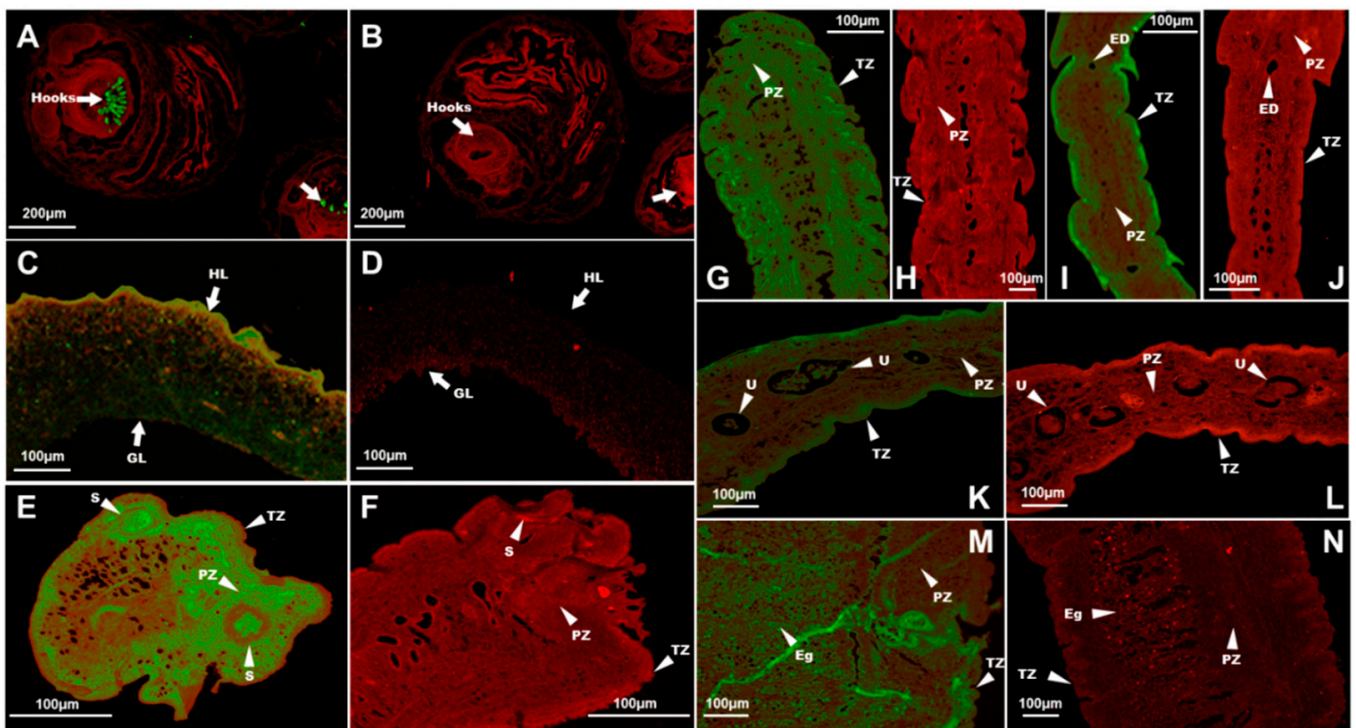

Figure 7. Immunofluorescence localization of TmAnxB3 in T. multiceps. Against the red background, the green fluorescent color shows the location of the native TmAnxB3 protein: (A) protoscolex with positive serum; (B) protoscolex with negative serum; (C) cyst wall with positive serum; (D) cyst wall with negative serum; GL, germinal layer; HL, horny layer; (E) scolex with positive serum; (F) scolex with negative serum; $(\mathbf{G})$ neck with positive serum; $(\mathbf{H})$ neck with negative serum; $(\mathbf{I})$ immature proglottids with positive serum; (J) immature proglottids with negative serum; (K) mature proglottids with positive serum; (L) mature proglottids with negative serum; $(\mathbf{M})$ gravid proglottids with positive serum; and (N) gravid proglottids with negative serum. Abbreviations: S, suckers; GL, germinal layer; HL, horny layer; TZ, tegument zone; PZ, parenchymatous zone; ED, excretory duct; U, Uterus; Eg, eggs. 


\subsection{Transcriptional Profiles of Taenia multiceps Annexins}

The qRT-PCR results showed that TmAnxB2 was transcribed in both adults and larvae. As for the expression of TmAnxB2 in adults, no significant differences $(p>0.05)$ were observed in transcriptional level between the segments. However, the relative amount of TmAnxB2 mRNA in immature proglottids was 1.6-, 3- and 3.6-fold higher compared to scolex, mature proglottids and gravid proglottids, respectively. Meanwhile, in the larval stage, the mRNA levels of TmAnxB2 were highest in oncosphere, 2.78-fold higher than in protoscolex and 5-20 times higher than in the adults $(p<0.05$; Figure 8). These results suggest that TmAnxB2 may play important roles in parasite development and host invasion of T. multiceps oncospheres.
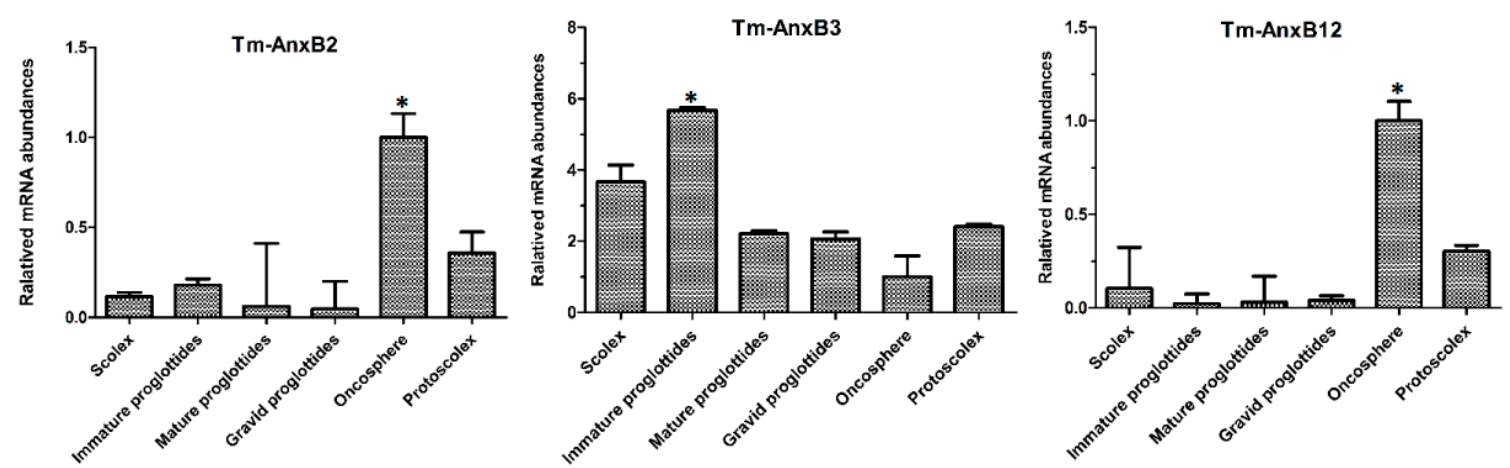

Figure 8. Differential transcription levels of TmAnxB2, TmAnxB3 and TmAnxB12 in different proglottids and developmental stages of T. multiceps. Levels of expression were determined using the ${ }^{\Delta \Delta} \mathrm{Ct}$ method. Data are represented as the means $\pm \mathrm{SD}(n=3)$. Asterisks indicate fold changes that were statistically significant at $p<0.05$.

Additionally, the TmAnxB3 gene was transcribed across all developmental stages of T. multiceps, with the highest expression in immature proglottids, 1.4-5.1-fold higher compared to other developmental stages or segments $(p<0.05)$. Interestingly, relative TmAnxB3 mRNA levels increased gradually from oncospheres to adults, suggesting that TmAnxB3 may be critical for T. multiceps development from larvae to adult stages (Figure 8). Similarly, TmAnxB12 gene expression was highest in oncospheres of T. multiceps, 3-50 times higher than in other stages and tissues $(p<0.05)$. Furthermore, relative TmAnxB12 mRNA levels in protoscolex were 3-15 times higher than in proglottids $(p<0.05$, Figure 8). These findings indicate that TmAnxB12 may have an important role in growth and development of $T$. multiceps, and possibly the host invasion.

\section{Discussion}

Annexins are a multigene protein family with typical conserved sequence characteristics. Members of this family have four homologous repeat domains, each of $\approx 70$ amino acid residues, and these domains generally include a typical K-G-X-G-T motif and a typical annexin type II binding site [20]. Structural prediction of TmAnxB2, TmAnxB3 and TmAnxB12 revealed that all these share the typical characteristics of annexin family members. However, TmAnxB2 appears to lack the calcium binding sites, which are replaced by a unique KGD motif in some repeats. This feature has been reported in other cestode homologs such as Ts-AnxB2, Eg-Anx, Em-Anx, Ht-Anx and Mc-Anx [21-23]. Nevertheless, it is worth noting that such structure was just modeled by referring to the available crystal structure of S. mansoni annexin and needs to be examined further in future. Moreover, the ML-based phylogeny placed these three annexin homologs of T. multiceps in different groups, in which TmAnxB2 and TmAnxB3 clustered with annexin B2 and B3 of T. solium, respectively. Interestingly, in a recent genome-based study, Cantacessi and colleagues showed that among six distinct annexin clades (I-VI) found across parasitic plathyhelminthes and nematodes, T. solium annexin B2 and B3 were positioned in Clade I [20]. Therefore, it is reasonable to infer that TmAnxB2 and TmAnxB3 of T. multiceps might 
also be positioned under such clade. Previous reports have demonstrated that protein phosphorylation plays a key role in parasitic flatworms, including coordination of differentiation of cells and organs and mediation of signal transduction [24-27]. Our predictions revealed numerous phosphorylation sites in TmAnxB2, TmAnxB3 and TmAnxB12, suggesting their potential implication in development and signal transduction. Moreover, by comparing the functional sites, we observed that TmAnxB3 has more protein kinase $C$ phosphorylation sites and casein kinase II phosphorylation sites compared to TmAnxB2 or TmAnxB12. Meanwhile, TmAnxB3 was most highly expressed in adult T. multiceps. Evidence from previous studies indicates that protein kinase $C$ and casein kinase II phosphorylation sites can play an important role in growth and development via catalytic phosphorylation of serine and threonine [24]. Similarly, our results suggest that TmAnxB3 may be an important protein having potential implication in growth and development in T. multiceps.

Annexins are calcium-dependent phospholipid binding proteins with calcium binding capacity that participate in various biological activities related to biofilm development via membrane phospholipid binding [15]. In parasites, Leishmania promastigotes bind annexin $\mathrm{V}$ to ensure the viability of promastigotes due to the lack of phosphatidylserine [28]. Meanwhile, annexin B1 of T. solium can induce calcium-dependent eosinophil apoptosis and diminish host immune responses [29-31]. Moreover, ANXB33 in E. granulosus is located in host inflammatory cells and fibroblasts, suggesting its potential involvement in reactions between hosts and parasites [21]. In the congeneric T. solium, previous studies showed that annexin B2 has a procoagulant effect in vitro, whereas annexin B1 binds to human eosinophils and generates a calcium current, thereby resisting attack by the host immune system $[32,33]$. In our present study, fluorescence immunolocalization showed that TmAnxB3 was expressed in both larvae and adults in T. multiceps, whereas TmAnxB2 was only expressed during the coenurus and oncosphere stages, suggesting that TmAnxB2 may be an important protein in the development of larvae, while TmAnxB3 may play a key role in the development of adult stages.

TmAnxB3 was mainly distributed in the tegument of each segment in T. multiceps, and was also widely distributed in the parenchymatous zone of the scolex and neck. These findings are consistent with the known distribution of annexins in E. granulosus, Clonorchis sinensis and S. mansoni [21,22,34]. The tegument of parasitic cestodes not only plays a key role in escaping the attack from host immune system, it is also an important organ for nutrient acquisition and signal transduction during long-term parasitic life [12]. In this study, TmAnxB3 was mainly distributed in the adult tegument, indicating its implication in nutrition and interaction between parasites and hosts. Furthermore, TmAnxB2 was mainly distributed in the cyst wall of T. multiceps, similar to AnxB1 in T. solium [30,35].

During parasitism, annexins can protect parasite tissues and help them escape attack from the host immune system [18]. Importantly, these proteins contribute to physiological functions in taeniidae in a tissue-specific manner [36,37]. For instance, AnxB30 of C. sinensis is transcribed at high levels in metacercaria and at lower levels in adults and eggs compared to other stages [34]. However, ANXB33 of E. granulosus is mainly transcribed in the germinal layer and protoscoleces, but not expressed in the cystic fluid or cyst wall [21]. In the present study, all three Tm annexins were expressed at relatively high levels in the metacestode stage, including oncosphere and coenurus stages. Notably, from coenurus to scolex, the relative transcription levels of TmAnxB2 and TmAnxB12 showed a downward trend, while the relative transcription of TmAnxB3 showed an upward trend. These findings suggest that TmAnxB3 may play a significant role in growth and development of adults. By contrast, TmAnxB2 and TmAnxB12 were transcribed at higher levels during the oncosphere period, and levels were statistically different from those in coenurus and scolex $(p<0.05)$, suggesting that these two proteins may play important roles in larvae of T. multiceps. Nevertheless, further studies are awaited to confirm these findings, but the recent report by Li et al. on genome of T. multiceps provides a comprehensive profile of annexins in this worm [1]. These enticing findings, to a certain extent, suggest that more annexin genes should be considered for elucidating their common and/or specific roles in different tissues (e.g., scolex-neck proglottids, immature-mature proglottids and gravid proglottids) and developmental stages (e.g., oncospheres, protoscolexes and adults) of T. multiceps. In addition, 
the development of tapeworms is dominated by the development of segments, hence newly emerged segments are closer to the scolex [38,39]. Similar to other metazoans, protein phosphorylation in tapeworms coordinates the differentiation of cells and organs [24], and the numerous phosphorylation sites in TmAnxB2, TmAnxB3 and TmAnxB12 observed in our study are consistent with this, suggesting the potential implication of annexins in regulating the important biological functions i.e., growth and development in T. multiceps.

\section{Conclusions}

In this study, the full-length cDNAs encoding three homologous annexins (TmAnxB2, TmAnxB3 and TmAnxB12) were identified and characterized in T. multiceps. From these, TmAnxB2 and TmAnxB3 proteins were localized in the oncosphere, protoscolex, scolex, immature proglottids, mature proglottids, and gravid proglottids, as demonstrated by fluorescence immunohistochemistry. Furthermore, TmAnxB2 was demonstrated to possibly play an important role in the larval stage of T. multiceps, while TmAnxB3 appears to be more important in adults. Taken together, these enticing findings of our study provide the reasonable basis for future studies focusing on exploration of potential functions of annexins in T. multiceps.

Author Contributions: Conceptualization, G.Y. and Y.X.; Methodology, Y.X. and C.G.; Software, C.G. and N.W.; Validation, C.G., Y.X., N.W. and Y.L.; Formal Analysis, C.G. and Y.X.; Resources, G.Y., X.P. and W.L.; Data Curation, C.G., Y.X., J.Z., X.Z., C.A. and X.G.; Writing-Original Draft Preparation, C.G.; Writing-Review and Editing, G.Y., Y.X. and C.A.; Supervision, G.Y.; Project Administration, G.Y.; and Funding Acquisition, G.Y.

Funding: This research was funded by Sichuan Science and Technology Department Project (2015NZ0041).

Conflicts of Interest: The authors declare no conflict of interest.

\section{References}

1. Li, W.; Liu, B.; Yang, Y.; Ren, Y.; Wang, S.; Liu, C.; Zhang, N.; Qu, Z.; Yang, W.; Zhang, Y.; et al. The genome of tapeworm Taenia multiceps sheds light on understanding parasitic mechanism and control of coenurosis disease. DNA Res. 2018, in press. [CrossRef] [PubMed]

2. Li, W.H.; Jia, W.Z.; Qu, Z.G.; Xie, Z.Z.; Luo, J.X.; Yin, H.; Sun, X.L.; Blaga, R.; Fu, B.Q. Molecular characterization of Taenia multiceps isolates from Gansu province, China by sequencing of mitochondrial cytochrome c oxidase subunit 1. Korean J. Parasitol. 2013, 51, 197-201. [CrossRef] [PubMed]

3. Huang, X.; Xu, J.; Wang, Y.; Guo, C.; Chen, L.; Gu, X.; Lai, W.; Peng, X.; Yang, G. GP50 as a promising early diagnostic antigen for Taenia multiceps infection in goats by indirect ELISA. Parasit. Vectors 2016, 9, 618. [CrossRef] [PubMed]

4. Wang, N.; Wang, Y.; Ye, Q.; Yang, Y.; Wan, J.; Guo, C.; Zhan, J.; Gu, X.; Lai, W.; Xie, Y.; et al. Development of a direct PCR assay to detect Taenia multiceps eggs isolated from dog feces. Vet. Parasitol. 2018, 251, 7-11. [CrossRef] [PubMed]

5. Oryan, A.; Moazeni, M.; Amrabadi, O.; Akbari, M.; Sharifiyazdi, H. Comparison of distribution pattern, pathogenesis and molecular characteristics of larval stages of Taenia multiceps in sheep and goats. Small Rumin. Res. 2015, 132, 44-49. [CrossRef]

6. Christodoulopoulos, G.; Dinkel, A.; Romig, T.; Ebi, D.; Mackenstedt, U.; Loos-Frank, B. Cerebral and non-cerebral coenurosis: On the genotypic and phenotypic diversity of Taenia multiceps. Parasitol. Res. 2016, 115, 4543-4558. [CrossRef] [PubMed]

7. Guo, C.; Wang, Y.; Huang, X.; Wang, N.; Yan, M.; He, R.; Gu, X.; Xie, Y.; Lai, W.; Jing, B.; et al. Molecular cloing and bioinformatics analysis of lactate dehydrogenase from Taenia multiceps. Parasitol. Res. 2017, 116, 2845-2852. [CrossRef] [PubMed]

8. Nie, H.M.; Xie, Y.; Fu, Y.; Yang, Y.D.; Gu, X.B.; Wang, S.X.; Peng, X.; Lai, W.M.; Peng, X.R.; Yang, G.Y. Cloning and characterization of the fatty acid-binding protein gene from the protoscolex of Taenia multiceps. Parasitol. Res. 2013, 112, 1833-1839. [CrossRef] [PubMed]

9. Amer, S.; ElKhatam, A.; Fukuda, Y.; Bakr, LI.; Zidan, S.; Elsify, A.; Mohamed, M.A.; Tada, C.; Nakai, Y. Clinical, pathological, and molecular data concerning Coenurus cerebralis in sheep in Egypt. Data Brief 2018, 16, 1-9. [CrossRef] [PubMed] 
10. Dalimi, A.; Sattari, A.; Motamedi, G. A study on intestinal helminthes of dogs, foxes and jackals in the western part of Iran. Vet. Parasitol. 2006, 142, 129-133. [CrossRef] [PubMed]

11. Aliye, J.; Deressa, F.B. Prevalence and economic importance of Coenurus Cerebralis in sheep and goats in and around "Legahida" district of bale zone, south eastern Ethiopia. Comp. Clin. Pathol. 2017, 26, 483-492. [CrossRef]

12. Wu, X.; Fu, Y.; Yang, D.; Xie, Y.; Zhang, R.; Zheng, W.; Nie, H.; Yan, N.; Wang, N.; Wang, J. Identification of neglected cestode Taenia multiceps microRNAs by illumina sequencing and bioinformatic analysis. BMC Vet. Res. 2013, 9, 162. [CrossRef] [PubMed]

13. Collomb, J.; Machouart, M.; Biava, MF.; Brizion, M.; Montagne, K.; Plénat, F.; Fortier, B. Contribution of $\mathrm{NADH}$ dehydrogenase subunit I and cytochrome c oxidase subunit I sequences toward identifying a case of human coenuriasis in France. J. Parasitol. 2007, 93, 934-937. [CrossRef] [PubMed]

14. Wang, Y.; Nie, H.; Gu, X.; Wang, T.; Huang, X.; Chen, L.; Lai, W.; Peng, X.; Yang, G. An ELISA using recombinant TmHSP70 for the diagnosis of Taenia multiceps infections in goats. Vet. Parasitol. 2015, 212, 469-472. [CrossRef] [PubMed]

15. Rescher, U.; Gerke, V. Annexins-Unique membrane binding proteins with diverse functions. J. Cell Sci. 2004, 117, 2631-2639. [CrossRef] [PubMed]

16. Chander, A.; Chen, X.L.; Naidu, D.G. A role for diacylglycerol in annexin a 7-mediated fusion of lung lamellar bodies. Biochim. Biophys. Acta 2007, 1771, 1308-1318. [CrossRef] [PubMed]

17. Mortimer, J.C.; Laohavisit, A.; Macpherson, N.; Webb, A.; Brownlee, C.; Battey, N.H.; Davies, J.M. Annexins: Multifunctional components of growth and adaptation. J. Exp. Bot. 2008, 59, 533. [CrossRef] [PubMed]

18. Hofmann, A.; Osman, A.; Leow, C.Y.; Driguez, P.; McManus, D.P.; Jones, M.K. Parasite annexins-New molecules with potential for drug and vaccine development. Bioessays 2010, 32, 967-976. [CrossRef] [PubMed]

19. Mu, J.; Yang, Y.D.; Yang, G.Y.; Wang, Y.W.; An, X.X.; Gu, X.B.; Bian, Y.; Fu, Y.; Wang, S.X. Biological characteristics of Taenia multiceps Sichuan strain. Chin. Vet. Sci. 2010, 36, 327-330.

20. Cantacessi, C.; Seddon, J.M.; Miller, T.L.; Leow, C.Y.; Thomas, L.; Mason, L.; Willis, C.; Walker, G.; Loukas, A.; Gasser, R.B. A genome-wide analysis of annexins from parasitic organisms and their vectors. Sci. Rep. 2013, 3, 2893. [CrossRef] [PubMed]

21. Song, X.; Hu, D.; Zhong, X.; Wang, N.; Gu, X.; Wang, T.; Peng, X.; Yang, G. Characterization of a secretory annexin in Echinococcus granulosus. Am. J. Trop. Med. Hyg. 2016, 94, 626-633. [CrossRef] [PubMed]

22. Tararam, C.A.; Farias, L.P.; Wilson, R.A.; Leite, L.C. Schistosoma mansoni annexin 2: Molecular characterization and immunolocalization. Exp. Parasitol. 2010, 126, 146-155. [CrossRef] [PubMed]

23. Morgan, R.O.; Martin-Almedina, S.; Iglesias, J.M.; Gonzalez-Florez, M.I.; Fernandez, M.P. Evolutionary perspective on annexin calcium-binding domains. Biochim. Biophys. Acta 2004, 1742, 133-140. [CrossRef] [PubMed]

24. Dissous, C.; Khayath, N.; Vicogne, J.; Capron, M. Growth factor receptors in helminth parasites: Signalling and host-parasite relationships. FEBS Lett. 2006, 580, 2968-2975. [CrossRef] [PubMed]

25. Bahia, D.; Andrade, L.F.; Ludolf, F.; Mortara, R.A.; Oliveira, G. Protein tyrosine kinases in Schistosoma mansoni. Mem. Inst. Oswaldo Cruz 2006, 101, 137-143. [CrossRef] [PubMed]

26. Brehm, K. The role of evolutionarily conserved signalling systems in Echinococcus multilocularis development and host-parasite interaction. Med. Microbiol. Immunol. 2010, 199, 247-259. [CrossRef] [PubMed]

27. Cheetham, G.M. Novel protein kinases and molecular mechanisms of autoinhibition. Curr. Opin. Struct. Biol. 2004, 14, 700-705. [CrossRef] [PubMed]

28. Weingärtner, A.; Kemmer, G.; Müller, F.D.; Zampieri, R.A.; Gonzaga dos Santos, M.; Schiller, J.; Pomorski, T.G. Leishmania promastigotes lack phosphatidylserine but bind annexin $\mathrm{V}$ upon permeabilization or miltefosine treatment. PLoS ONE 2012, 7, e42070. [CrossRef] [PubMed]

29. Solano, S.; Cortés, I.M.; Copitin, N.I.; Tato, P.; Molinari, J.L. Lymphocyte apoptosis in the inflammatory reaction around Taenia solium metacestodes in porcine cysticercosis. Vet. Parasitol. 2006, 140, 171-176. [CrossRef] [PubMed]

30. Gao, Y.J.; Yan, H.L.; Ding, F.X.; Lu, Y.M.; Sun, S.H. Annexin B1 at the host-parasite interface of the Taenia solium cysticercus: Secreted and associated with inflammatory reaction. Acta Trop. 2007, 101, 192-199. [CrossRef] [PubMed] 
31. Patel, N.; Hamamouch, N.; Li, C.; Hewezi, T.; Hussey, R.S.; Baum, T.J.; Mitchum, M.G.; Davis, E.L. A nematode effector protein similar to annexins in host plants. J. Exp. Bot. 2010, 61, 235-248. [CrossRef] [PubMed]

32. Wang, K.; Guo, Y.; Li, K.; Lu, Y.; Zhang, Y.; Sun, S.; Yan, H.; Zhang, S. Molecular characterization and anticoagulant activity of a novel annexin derived from the Taenia solium. Acta Trop. 2006, 99, 165-172. [CrossRef] [PubMed]

33. Yan, H.L.; Xue, G.; Mei, Q.; Ding, F.X.; Wang, Y.Z.; Sun, S.H. Calcium-dependent proapoptotic effect of Taenia solium metacestodes annexin B1 on human eosinophils: A novel strategy to prevent host immune response. Int. J. Biochem. Cell. Biol. 2008, 40, 2151-2163. [CrossRef] [PubMed]

34. He, L.; Ren, M.; Chen, X.; Wang, X.; Li, S.; Lin, J.; Liang, C.; Liang, P.; Hu, Y.; Lei, H.; et al. Biochemical and immunological characterization of annexin B30 from Clonorchis sinensis excretory/secretory products. Parasitol. Res. 2014, 113, 2743-2755. [CrossRef] [PubMed]

35. Wei, C.X.; Li, T.L.; Suo, Y.L. Advances in annexin of Taeniidae. Contemp. Anim. Husb. 2015, 3-4. [CrossRef]

36. Dai, J.L.; Huang, J.; Liao, X.J.; Li, B.; Wang, Y. The expression and purification of the annexins B2 gene in Taenia saginata asiatica and the immunologic analysis of the recombinant proteins. J. Xi'an Jiaotong Univ. 2010, 30, 696-698.

37. Wang, Z.W.; Han, H.Y.; Huang, B. Four alctium signaling proteins and their preliminary studies in parasitology. Chin. J. Anim. Infect. Dis. 2015, 23, 78-86.

38. Wang, Z.X.; Zhang, Y.Y.; Lu, P.P.; Ma, X.; Wang, Z.R.; Bo, X.W. The role of wnt signaling pathway in the flatworm. Chin. J. Anim. Vet. Sci. 2017, 48, 207-213.

39. Wang, Z.R.; Zhang, Y.Y.; Bo, X.W.; Xu, X.P.; Xu, C.S. Differential expression of six wnt gene family members in Echinococcus granulosus protoscoleces and adult worms. Chin. J. Parasitol. Parasit. Dis. 2016, 34, 552-557.

(C) 2018 by the authors. Licensee MDPI, Basel, Switzerland. This article is an open access article distributed under the terms and conditions of the Creative Commons Attribution (CC BY) license (http:/ / creativecommons.org/licenses/by/4.0/). 